\title{
Application of X-ray micro-computed tomography on high-speed cavitating diesel fuel flows
}

\author{
N. Mitroglou ${ }^{1}$ (D) M. Lorenzi ${ }^{1} \cdot$ M. Santini ${ }^{2} \cdot$ M. Gavaises ${ }^{1}$
}

Received: 8 August 2016 / Revised: 23 September 2016 / Accepted: 25 September 2016 / Published online: 4 November 2016 (C) The Author(s) 2016. This article is published with open access at Springerlink.com

\begin{abstract}
The flow inside a purpose built enlarged singleorifice nozzle replica is quantified using time-averaged $\mathrm{X}$-ray micro-computed tomography (micro-CT) and highspeed shadowgraphy. Results have been obtained at Reynolds and cavitation numbers similar to those of real-size injectors. Good agreement for the cavitation extent inside the orifice is found between the micro-CT and the corresponding temporal mean 2D cavitation image, as captured by the high-speed camera. However, the internal 3D structure of the developing cavitation cloud reveals a hollow vapour cloud ring formed at the hole entrance and extending only at the lower part of the hole due to the asymmetric flow entry. Moreover, the cavitation volume fraction exhibits a significant gradient along the orifice volume. The cavitation number and the needle valve lift seem to be the most influential operating parameters, while the Reynolds number seems to have only small effect for the range of values tested. Overall, the study demonstrates that use of microCT can be a reliable tool for cavitation in nozzle orifices operating under nominal steady-state conditions.
\end{abstract}

\section{Introduction}

The ever more stringent emission regulations of directinjection diesel engines, imposed by the regulatory boards worldwide, have triggered the race of improving the

M. Gavaises

m.gavaises@city.ac.uk

1 School of Mathematics Computer Science and Engineering, City University London, London EC1V OHB, UK

2 Department of Engineering, University of Bergamo, 24129 Bergamo, Italy efficiency of such engines. Among others, the fuel injection equipment (FIE) is a key component for improving the efficiency of environmentally friendly engines. Recent advances dictate the use of extra high injection pressures, of the order of 3000 bar and tiny flow passages (injection holes) of the order of $100-250 \mu \mathrm{m}$ in diameter, for improved fuel atomisation and combustion efficiency. Under these circumstances, cavitation has been found to develop inside fuel injection nozzles at the early studies of Badock et al. (1999), Bergwerk (1959), Chaves et al. (1995) and Nurick (1976), followed by Arcoumanis et al. (2000), Blessing et al. (2003), Mitroglou et al. (2014) and Roth et al. (2002) in more realistic real-size nozzle geometries offering optical access; equally helpful studies performed in transparent enlarged nozzle replicas [selectively (Andriotis et al. 2008; Arcoumanis et al. 2000; Miranda et al. 2003; Mitroglou and Gavaises 2013; Powell et al. 2000)] also indicate that cavitation plays an increasingly significant role in the nozzle's internal flow structure and development. Cavitation inside an injection hole is believed to enhance spray atomisation, either directly through the implosion of cavitation bubbles or indirectly because it increases turbulence in the nozzle flow (Badock et al. 1999; Walther 2002); unfortunately, under certain circumstances induces erosion (Dular and Petkovšek 2015; Koukouvinis et al. 2016) that may lead to catastrophic failures. Moreover, cavitation inside the injection holes promotes shot-toshot spray instabilities (Mitroglou et al. 2011, 2012; Suh and Lee 2008), which, in turn, are responsible for poor combustion efficiency and increased emissions (Hayashi et al. 2013). Two distinct macroscopic forms of cavitation have been identified, which are referred to as geometryinduced and vortex or 'string' cavitation. The former can be, partially or totally, suppressed by appropriate design of the inlet hole curvature and non-cylindrical injection hole 
shape (Soteriou et al. 2006), for sufficiently high positions of the needle valve (i.e. open); this is now the standard practice in manufacturing automotive diesel injector nozzles (Potz et al. 2000); still, some cavitation may be needed as it is thought to make nozzles more resistant against deposit formation (Tang et al. 2009). Cavitation is also known to decrease the nozzle flow rate (Soteriou et al. 1995) and lead in some cases (but not always) to flow choking (Payri et al. 2006). On the other hand, vortex-induced cavitation and turbulent flow in a fuel injector nozzle may lead to complex structures which remain not well-understood.

Nozzle cavitation has been traditionally studied using visualisation techniques; in some of the relevant early studies, parts of a nozzle were replaced with transparent replicas (i.e. single injection hole), allowing unobstructed visible access (Chaves et al. 1995; Roth et al. 2005; Winklhofer et al. 2001). Many investigations have been performed on transparent, enlarged full nozzle replicas; due to the significantly lower injection pressures utilised in such enlarged models (Gavaises et al. 2009; Mitroglou and Gavaises 2013; Soteriou et al. 2006), dimensionless similarity based on the cavitation $(\mathrm{CN})$ and Reynolds $(R e)$ numbers has been adopted. Parallel to those activities, attempts were also made to manufacture real-size exact replicas of diesel nozzles from transparent materials that are able of withstanding pressures in the range of 500-2000 bar, which are similar to those in real injections. Such successful studies have been presented in Blessing et al. (2003), Hayashi et al. (2013), Jollet et al. (2013), Mitroglou et al. (2011, 2014) and Reid et al. $(2010,2014)$ and have confirmed the observations reported from the enlarged transparent models. In most cases, there is evidence of the interrelated geometric-induced and vortex cavitation. Moreover, string cavitation owes its existence either to vortices that cavitate or to sources of vapour, or pre-existing nuclei inside the nozzle that grow when trapped inside flow vortices. All observations so far have confirmed that the developed multi-phase flow field is complex and exhibits a transient behaviour, even at simplified single-orifice geometries.

High-speed visualisation applied on all aforementioned investigations have succeeded in capturing in detail the transient flow behaviour; however, they exhibit certain drawbacks due to the fact that light scatters and refracts strongly on liquid-vapour interfaces present inside the flow channel. Single scattering, and more severely multiple scattering, reduces, or totally blocks, light transmission, even at very low vapour fractions; the latter degrades the ability of optical techniques to provide quantitative measurements of fluid density, especially in optically dense multiphase flow fields and even increases the uncertainty of the produced qualitative representations.

Despite the opacity of multiphase flow systems, quantitative experiments are necessary for the deeper understanding of the flow physics; in this direction, several research groups worldwide have developed other non-invasive measurement techniques. As stated in Dudukovic (2000), a multiphase flow experimental system should have: (1) high spatial and temporal resolution for local phase fraction measurements, as well as velocity field measurements of all phases, and (2) the capability to provide instantaneous and time history snapshots of the flow. Note, however, that a single experimental system that can satisfy all these needs does not currently exist (Heindel 2011). Despite the recent advances in laser diagnostics, such as ballistic imaging or structured illumination to mitigate the multiple scattering effects (Berrocal et al. 2008; Linne et al. 2006), their ability to measure fluid density is still limited (Leick et al. 2011). Moreover, flows with significant refractive index gradients, such as liquid diesel and vapour, exhibit beam-steering effects that interfere with optical diagnostics by creating uncertainty in the location of the volume under observation (Heindel 2011). Invasive measurement techniques, such as probes, interfere and alter the internal flow field that is under investigation and thus, they cannot be used with the small orifices utilised with fuel injection equipment.

Multiple non-invasive quantitative flow visualisation techniques have been reviewed in the literature over the years (Chaouki et al. 1997; Dudukovic 2000; Duke 2015; Heindel 2011; Kastengren and Powell 2014; Yates et al. 2002). One of the first applications of $X$-ray imaging for visualisation of air bubbles in gas-solid fluidised beds was performed in the 1950s and 1960s (Grohse 1955; Rowe and Partridge 1997). A variety of tomographic, or 2-D radiographic, X-ray techniques have been developed, where tomography refers to the cross-sectional imaging of a system from either transmission or reflection data collected by illumination of the flow system from many different angles (Kak and Slaney 2001), while radiography refers to single-angle collection data. In X-ray tomography, unlike soft-field methods (Marashdeh et al. 2008), the field lines of the measured property (e.g., X-ray attenuation) are not influenced by material density changes outside the path of a single X-ray. The latter simplifies reconstruction; however, due to the current technology of detection systems and X-ray source flux, data acquisition is typically slow, in relation to cavitation time-scales, for a laboratory-scale micro-tomography facility. On the contrary, synchrotron $\mathrm{X}$-ray sources provide a far greater degree of brilliance than laboratory-scale sources, permitting a much greater scope for advanced X-ray diagnostics. Synchrotron sources have existed for many years, but have until recently found relatively little usage by the fuel injection community (Duke et al. 2013, 2014, 2015a; Kastengren et al. 2012; Moon et al. 2015; Powell et al. 2000; Wang et al. 2008). Nevertheless, laboratory-scale X-ray sources have improved characteristics in recent years, featuring small spot-sizes, 
which if coupled with developments in detector technology, provide adequate spatial resolution for multiphase flow applications.

One of the first attempts to apply X-ray CT measurements on multiphase flows is dated back at 1983 (Ikeda et al. 1983); the technique was applied on a steady-state U-shaped tube air-water flow system. Measured void fractions were compared against results of intrusive techniques and demonstrated good agreement. Recent examples of application of $\mathrm{X}$-ray techniques on cavitating flows are found in CoutierDelgosha et al. (2006) and Stutz and Legoupil (2003); tube source X-rays, with relatively low photon fluxes, have been used to provide vapour volume fraction measurements of sheet cavitation over a hydrofoil inside a two-dimensional channel flow. Additionally, recent investigations on cavitating flow inside cylindrical flow channels is found in Bauer et al. (2012); a medical CT scanner was utilised for providing measurements inside an enlarged cavitating pipe flow ( $D=20 \mathrm{~mm}$ ) using water as the working fluid. The reconstructed void fraction along the pipe length revealed the formation of water vapour at the channel walls, for the first half of the hole length (forming a liquid core), and also a weak vapour accumulation around the channel axis (core of the flow) at the second half of the channel length. The former, which had been observed previously using visualisation techniques, was an expected behaviour; however, the latter, which had not been captured by conventional visualisation techniques, was an unexpected flow feature not previously observed. Similarly, a highly tuned medical X-ray source was used in Ganesh et al. (2015), Mäkiharju et al. (2013) and Sun et al. (2015) to study cavitation in the wake of bluff bodies and two-dimensional wedges inside a water tunnel. As demonstrated, low void fraction regions in partial cavities could provide conditions necessary for the existence of bubbly shocks, which act as a dominant mechanism of shedding of partial cavities under certain conditions. The latter behaviour was verified using X-ray densitometry at frame rates reaching $1 \mathrm{kHz}$, an acquisition speed that enabled the capture of dynamics of bubbly shock fronts with and without the presence of an obstacle. Finally, time-resolved and mean X-ray densitometry-based vapour volume fraction of the span-wise and plan view (for the case of a 2-D wedge) averaged flow field have been related to the vortex dynamics in order to explain vapour production in such transient flow dynamics.

Tube source X-rays have a large wavelength spread and a divergent beam and can provide high spatial resolution over long acquisition times; time-resolved measurements can be obtained at reduced spatial resolution; however, it is difficult to satisfy both requirements simultaneously. Such problems are overcome by the use of focused synchrotron $\mathrm{X}$-rays that offer an advantageous combination of temporal and spatial resolution due to high photon flux; however, the main drawback is the limitation of focus in a relatively small area (Duke et al. 2013). Nevertheless, relevant studies have been performed in Battistoni et al. (2015) and Duke et al. (2013, 2014, 2015b), where multiphase flow inside a cavitating channel has been studied and compared to computational fluid dynamics (CFD) results. Researchers performed an X-ray radiography experiment on a model nozzle of $500 \mu \mathrm{m}$ in diameter. They exploited raster scan radiography and achieved a spatial resolution of $5 \mu \mathrm{m}$ and a temporal resolution of $3.6 \mu \mathrm{s}$. Their experimental test-rig was able of delivering high $\mathrm{CN}$ at low discharge pressures, and $R e$ of the order of $15 \mathrm{k}$. Vapour structures were found to be relatively steady, as time-resolved measurements indicated that root-mean square (RMS) fluctuations did not exceed $1 \%$ of the mean values. The spectral content of cavitation was found to be concentrated at small Strouhal numbers, of the order of 0.001-0.1, suggesting steady cavitation inception and mixing processes without large-scale fluctuations. Extensive vapour regions at the nozzle centreline, where cavitation is not expected to occur, were identified, similar to Bauer et al. (2012); however, the authors reported that this could be due to dissolved gas present in the fuel that comes out of solution if the static pressure at that region is low. Similar studies involving synchrotron sources have also been applied on dense, high-pressure diesel/gasoline sprays, as described in Moon et al. (2010, 2015), where an ultrafast X-ray imaging technique has been applied. All recent advances in synchrotron X-rays and relevant imaging techniques are well described in Duke et al. (2015a, b), Duke (2015) and Kastengren and Powell (2014).

The present investigation exploits the increased spatial resolution of a cone-beam CT system (Santini et al. 2013) to quantitatively measure mean, three-dimensional vapour volume fraction inside a custom-design, scaled-up model channel flow that uses diesel as the working fluid. Due to the required long sampling times, the obtained mean vapour volume fractions are compared against ultra-high-speed flow visualisation results to qualitatively assess the dynamic behaviour of the flow. The study demonstrates the ability of a laboratory-scale X-ray source to provide the average, three-dimensional vapour volume fraction, and it is able to capture the effects of several flow parameters on cavitation structures present inside the custom injection hole.

\section{Methods}

The custom, purpose-built model features a non-symmetric flow arrangement that intends to mimic the flow patterns realised inside an orifice of a multi-hole diesel nozzle. 


\subsection{Nozzle geometry and high-pressure test-rig}

As illustrated in Fig. 1, the nozzle comprises a single injection hole $(D=3 \mathrm{~mm}, L=9.5 \mathrm{~mm})$, whose axis is at an offset to the main axis of symmetry of the device. The achieved asymmetry, combined with the non-symmetric needle, ensures a flow field that promotes cavitation mainly on one side of the orifice entry, enabling unobstructed view of the cavitation pocket created from any angle. This configuration is similar to the single-hole, high-pressure transparent design presented by Reid et al. (2010), which has operated at pressures up to 2000 bar. As presented in this study, the observed flow field inside and upstream of the orifice has many similarities in cavitation development to that of multi-hole diesel nozzles. The test-rig has been designed specifically for use inside the micro-CT facility developed at the University of Bergamo (Fig. 1). The needle is set at a fixed position, while the pressure difference across the orifice is also constant during one experimental case; tested values ranged from 10 to 40 bar. The hydraulic unit that drives the system is a commercial one, able of delivering a maximum flow rate of $40 \mathrm{l} / \mathrm{min}$. It was modified to accommodate a water-fuel heat exchanger equipped with a temperature control mechanism for accurate diesel fuel temperature control and monitor throughout the experimental campaign. The fuel temperature did not deviate more than $0.5^{\circ} \mathrm{C}$ from the set-point that was $40{ }^{\circ} \mathrm{C}$, regardless of the set pressure level (diesel properties at $40{ }^{\circ} \mathrm{C}$ : $\left.\rho=850 \mathrm{~kg} / \mathrm{m}^{3}, v \sim 3.9 \mathrm{~mm}^{2} / \mathrm{s}\right)$. The conditions presented in this study are listed in Table 1.
The material of the nozzle was selected in order to enhance the $\mathrm{X}$-ray signal to noise ratio. Various plastic and composite materials were evaluated that exhibit low X-ray attenuation, as opposed to metals; the polymer material PEEK (Polyether ether ketone) was selected, which exhibits low radiation attenuation and, at the same time, it has very appealing mechanical characteristics, necessary to withstand an inlet pressure of up to 55 bar and outlet pressure of up to 20 bar.

\subsection{X-ray computed tomography}

The advances in laboratory X-ray sources and detectors have allowed the progress and development of micro-CT. The process of CT imaging involves collection of projections (radiographies) from several hundreds of orientations (angles) of the X-ray intensity attenuated by the object of interest on an imaging device. Collected data (so-called sinograms) are then reconstructed using algorithms, such as filtered back-projection (also called FDK from the people who introduced it: Feldkamp-Davis-Kress) (Feldkamp et al. 1984), generating an image of the object crosssection. If the X-ray source is a cone-beam one (able of acquiring a $M \times N$ radiography, as opposed to a fan-beam type source that only acquires a $1 \times N$ radiography), then several slices are produced in a single scan, producing a $3 \mathrm{D}$ reconstructed image of the object. To reconstruct a reliable image, each projection should be acquired at stationary conditions; thus, it is essential for the object to be motionless at each orientation. This is not feasible in a

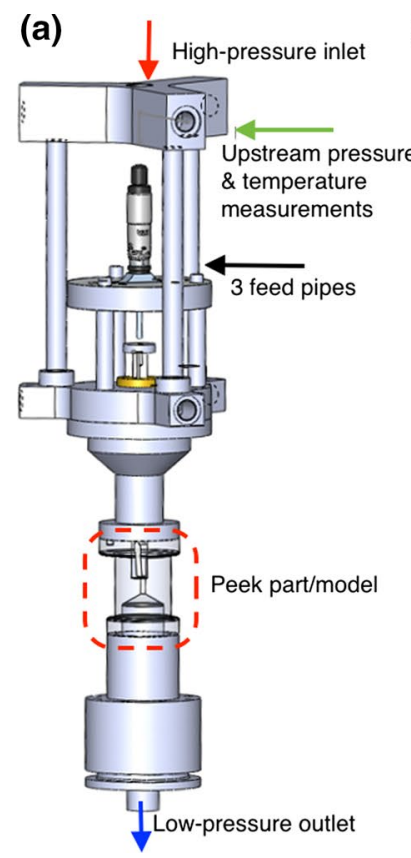

(b)

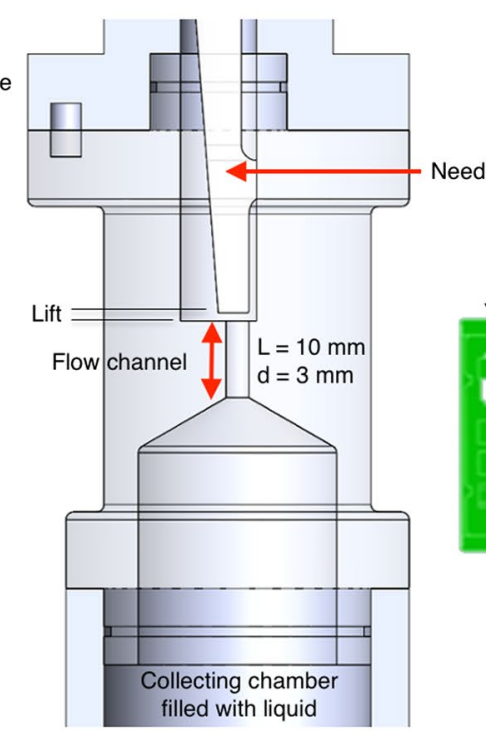

(c)

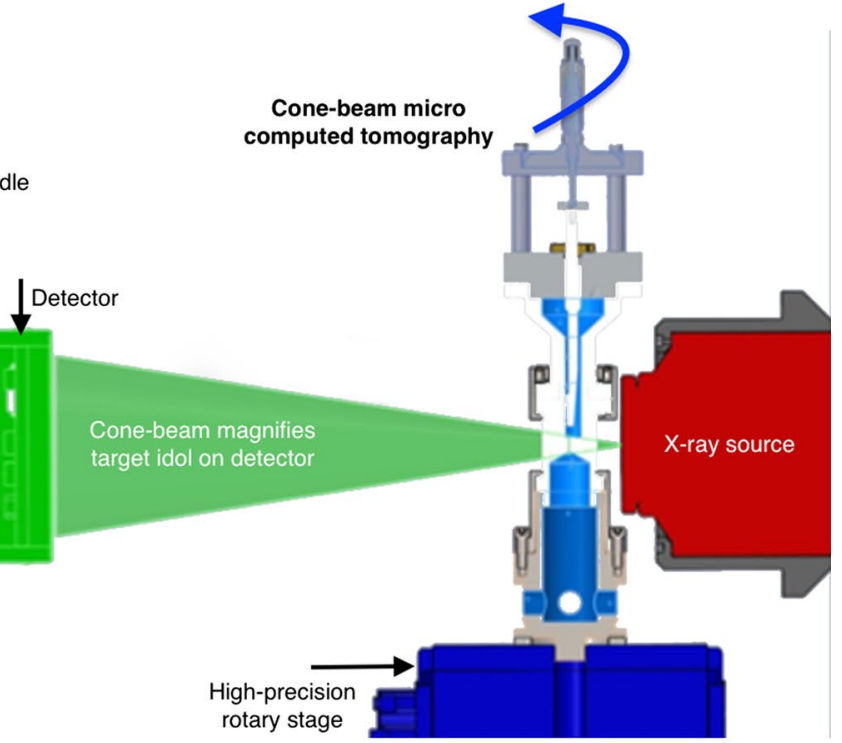

Fig. 1 a Overall test-rig set-up b flow channel model cross-section with dimensions and $\mathbf{c}$ tomography experimental set-up 
Table 1 Range of experimental conditions

\begin{tabular}{lllllll}
\hline & Range of operation & \multicolumn{7}{l}{ Experimental conditions presented } \\
\hline Needle lift (mm) & $0.5,1$ and 2 & 0.5 & 1 & 1 & 1 & 2 \\
$P_{\text {in }}$ (bar) & $10-60$ & 30 & 42 & 17 & 45 & 25 \\
$P_{\text {out }}$ (bar) & $5-20$ & 9.7 & 13.5 & 7 & 18 & 10 \\
Flow rate (1/min) & $14-30$ & 15.5 & 23.5 & 14.7 & 23.5 & 20 \\
$\mathrm{CN}^{\mathrm{a}}$ & $0.8-3.8$ & 2.1 & 2.1 & 1.5 & 1.5 & 1.5 \\
$R e$ & $30-67 \mathrm{k}$ & $36.5 \mathrm{k}$ & $55.5 \mathrm{k}$ & $34.6 \mathrm{k}$ & $55.5 \mathrm{k}$ & $47 \mathrm{k}$ \\
$C_{\mathrm{d}}$ & $0.5-0.78$ & 0.52 & 0.66 & 0.7 & 0.7 & 0.78 \\
Temp $\left({ }^{\circ} \mathrm{C}\right)$ & $40 \pm 0.5$ & & & & & \\
\hline
\end{tabular}

${ }^{\text {a }}$ Cavitation number: $\mathrm{CN}=\frac{P_{\text {inj }}-P_{\text {chamber }}}{P_{\text {chamber }}-P_{\text {vapour }}}$ transient multiphase flow environment, so each projection is acquired over a time scale that averages any signal fluctuation, which effectively results in averaging of the flow features observed. As shown in Ikeda et al. (1983), the result of reconstructed $\mathrm{CT}$ projections represents a time average of the varying quantities, when applied to dynamic flows; the latter is valid as long as the image acquisition time is much larger than the characteristic time scale of the variations.

The prototype micro-CT facility available at the University of Bergamo consists of a $160 \mathrm{kV} @ 400 \mu \mathrm{A}$ open type cone-beam $\mathrm{X}$-ray source, an air-bearing direct drive rotary stage and a $1944 \times 1536$ pixels flat panel CMOS detector characterised by a custom-matched scintillator plate in order to enhance the detection of low doses as used with low densities, such for the diesel fluid of interest. The X-ray facility is described in detail in Santini et al. (2013), where the validation of the technique for extraction and estimation of multiphase interfaces is also presented (Santini and Guilizzoni 2014; Santini et al. 2015). The spatial resolution has been adapted to obtain, in the reconstructed volume, an isotropic voxel size of $15 \mu \mathrm{m}$. This voxel dimension has been considered adequate to picture the cavitation phenomena of interest. For an accurate threedimensional reconstruction of the flow structures, tomographic projections were acquired over a full revolution of the test-nozzle $\left(360^{\circ}\right)$ every $0.6^{\circ}$ (600 projections). At each angular step, five projections were acquired and averaged to improve signal to noise ratio; therefore, the full scan time for each test case had been approximately $1 \mathrm{~h}$, since a projection acquisition time of $1 \mathrm{~s}$ had been used. During acquisition, no beam filtration was applied to the incoming X-rays produced by the source that was set-up to $60 \mathrm{kV}$ and $40.2 \mu \mathrm{A}$. The gathered radiographies of the flow inside the injection hole were corrected for any detector defects and normalised against a nominal X-ray intensity that corresponds to attenuation of X-rays through air. Following the application of the logarithmic absorption law, appropriate filters were used to enhance the boundaries of different density materials. Examples for both the normalised and filtered radiographies are illustrated in Fig. 2b, c; for the purposes of this study and due to the total absence of metal parts in the X-ray field of view, only a frequency ramp filter was applied and the result was rendered adequate for the subsequent application of the selected reconstruction algorithm. Therefore, the back-projection algorithm (Feldkamp et al. 1984) was translated in a custom-developed routine, where all collected radiographies were assembled in order to reconstruct the three-dimensional image of the flow field. Figure 2a illustrates a schematic of the conebeam computed tomography Cartesian systems; it is evident that the term "back-projection" refers to the process
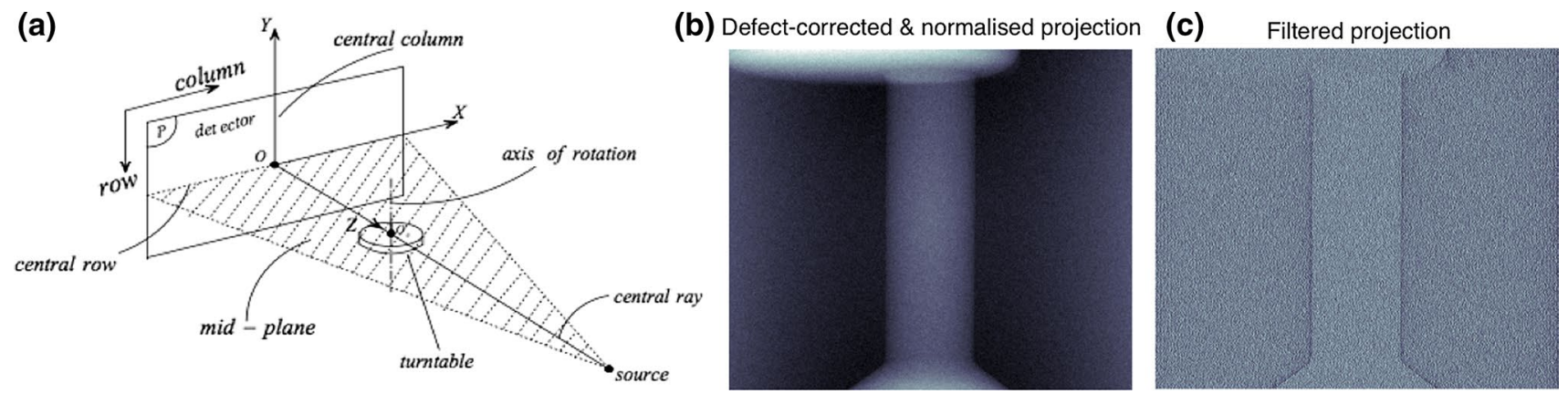

Fig. 2 a Schematic of cone-beam computed tomography Cartesian systems b normalised single radiography (projection) and $\mathbf{c}$ Ram-Lak filtered projection 
of back-projecting the acquired image on the detector plane (matrix of rows and columns), back to the Cartesian system of the original object (labelled "turntable"). In brief, the advantage of a cone-beam X-ray system is summarised in its ability to collect two-dimensional radiographies (as opposed to one-dimensional radiographies of the fan-beam system) of a magnified idol of the object in question. To achieve this, the setup takes advantage of the cone-beam angle and the ratio of distances of source-detector and source-object that determines the magnification factor.

Finally, a known source of errors, when reconstructing $\mathrm{X}$-ray CT images, are artefacts within the image. Common sources of artefacts are beam hardening, ring artefacts, and abrupt changes in density (Ketcham and Carlson 2001). Beam hardening is the most common artefact found in X-ray CT reconstructions that causes the edges of the scanned object to appear brighter than the centre, even for homogeneous materials. An effective way to reduce beam hardening that is more severe in metallic objects than plastics, is by filtering low-energy "soft" X-rays with metal filters. For this experimental campaign, no beam hardening filtration was planned mainly due to the absence of metallic parts and a possible reduction in the image contrast imposed by extra filtration. Instead, indirectly (by design), beam hardening was partly treated through the usage of beam filtration directly at the sample; i.e., the PEEK model acted as a beam filter surrounding the volume of interest (i.e. the injection hole), filtering the incoming $\mathrm{X}$-rays before they reach the channel. Ring artefacts, in the reconstructed image, are caused by non-uniform response of adjacent detector elements to changes in X-ray energy. These errors can be minimised through proper calibration; however, the nature of tomographic reconstruction will promote even subtle differences in adjacent detector elements to ring artefacts. The measures taken by the authors to reduce ring artefacts are summarised in (1) using as input for the reconstruction the bright and dark images for proper correction of the unevenness of the detector values without the sample being present; (2) calculating as accurately as possible the detector offset and tilt parameters to compensate for the non-nominal scanning geometry and introduced the pointwise calibration for the reconstructed slices to diminish also this kind of artefact. Nevertheless, such artefacts have been observed and are visible in Figs. 7 and 8 along the channel centreline. Finally, abrupt changes in system density result in sharp changes in intensity signals that lead to streaks in the reconstructed image, due to approximations in the reconstructed algorithms. The best way to minimise streaking is by optimised system design to minimise sharp density differences in the imaging region. Test cases of the current investigation that suffered streaks due to abrupt changes in density were only the $0.5-\mathrm{mm}$ needle lift cases, where the close vicinity of the metal needle to the injection hole created some inevitable problems. However, in order to discard any source of ambiguity and errors, we discarded the first $\sim 0.5 \mathrm{~mm}$ of the injection channel. Finally, it is essential to note that most of the known artefacts related to CT reconstructions are more pronounced in the case of metallic objects, as opposed to using softer materials, like plastic, that exhibit lower attenuation coefficients than metal.

\subsection{Post-processing}

Application of the filtered back-projection reconstruction process results in conversion of the intensity data in the sinogram files to CT numbers or CT values. These values correspond to the grey scale value in the resulting reconstructed image. It is important to highlight that during the $\mathrm{CT}$ reconstruction, the $\mathrm{CT}$ values usually map linearly to the effective attenuation coefficient of the material in each voxel (3D pixel) (2001). Hence, the variation in X-ray local attenuation is closely related to variations in local density (Du et al. 2008). This information is then used to determine local time-averaged phase fractions in a two-phase system.

Following the tomographic reconstruction of the flow channel, the obtained spatial X-ray attenuation coefficients are post-processed against two calibration sets in order to derive liquid fraction information per voxel; the post-processing procedure is described in Mitroglou et al. (2015) and a schematic of the procedure is illustrated in Fig. 3. In brief, the time-averaged liquid phase fraction in element $(i, j)$, equals the ratio of differences between the time-averaged CT value of the flowing system at location $(i, j), \mu_{i, j}$, minus the time-averaged CT value of $0 \%$ liquid phase $\left(\mu_{i, j}^{\mathrm{a} i \mathrm{r}}\right)$, over the difference of timeaveraged CT values of the two calibration sets, $\mu_{i, j}^{\mathrm{liq}}$ and $\mu_{i, j}^{\mathrm{air}}$. These correspond to time-averaged CT values of 100 and $0 \%$ liquid phase at location $(i, j)$, respectively. Finally, results refer to time-averaged liquid volume fraction information over the measurement period that is 1 hour, and the relevant error analysis showed that the followed post-processing procedure produced an error of $\sim 5 \%$. The latter is attributed to several facts, the most important being: the reconstruction technique, which is an approximate technique and the variation in X-ray intensity during a complete $360^{\circ}$ acquisition.

Following the extraction of liquid volume fraction information, it is evident that the data refer to the complete scanned volume. An extra post-processing procedure was followed that is a common practice in the X-ray synchrotron experimental community; see selectively (Duke et al. 2015a, b; Kastengren and Powell 2014; Lin et al. 2012). In brief, exploiting the uniformity of the Cartesian three-dimensional grid, the projected void along the channel length is calculated by a summation of the product of vapour volume fraction at each location $(i, j, k)$ multiplied by the corresponding voxel's volume; the sum is then 

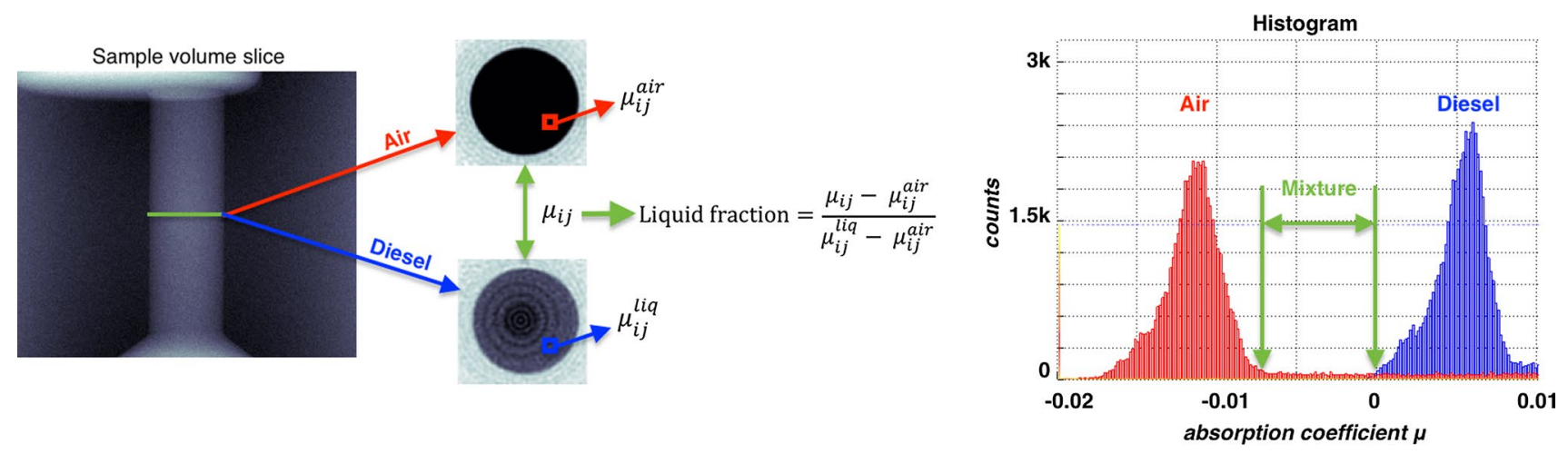

Fig. 3 Schematic of post-processing procedure followed to obtain liquid fraction of data sets, using the essential calibration sets

divided by the square of the cell grid size. The projected vapour volume fraction has length units and it effectively presents a line integral of the non-dimensional quantity ( $1-$ $\alpha_{\text {liq }}$ ) supplementary to liquid volume fraction (Duke et al. 2014) calculated in the previous processing step (Fig. 3).

\subsection{High-speed shadowgraphy}

A second model (manufacturing tolerance of $20 \mu \mathrm{m}$ ) was manufactured from Perspex that allowed high-speed shadowgraphy to be performed. As mentioned in earlier paragraphs, flow visualisation suffers severe light extinction even at very low vapour volume fractions; however, it enables ultra-high-speed photography that provides qualitative information on cavitation vapour topology. In the current study, shadowgraphy images of selected test cases were acquired at $80 \mathrm{k}$ frames per second. The collected images are then post-processed to extract statistical information on the topology of cavitation structures and their variations. The population used in this statistical analysis is $80 \mathrm{k}$ images per test case, which corresponds to real flow time of $1 \mathrm{~s}$. The derived mean and standard deviation images are presented alongside quantitative micro-CT measurements.

\section{Results}

The results presented in this section constitute a mix of quantitative X-ray time-averaged measurements and qualitative shadowgraphy statistical images. This helps the reader to complement time-averaged liquid volume fraction distributions inside the flow channel with ultra-fast images of the flow that capture the transient behaviour of the multi-phase flow system.

\subsection{Flow pattern}

Figure 4 is divided in two columns, illustrating high-speed shadowgraphy and quantitative X-ray time-averaged liquid volume fraction measurements at the left- and right-handside columns, respectively. As seen in the instantaneous high-speed image at the top-left corner of Fig. 4, where cavitation structures are imaged in dark shades and liquid in lighter shades, the vapour cloud occupies mainly the bottom side of the channel. As the flow comes from the lefthand side, the asymmetry of the flow test-rig directs the flow to enter the orifice from the bottom side thus, vapour on this side is extended at larger lengths inside the channel; on the contrary, at the top channel side cavitation collapses at relatively shorter lengths. Statistical information extracted from the shadowgraphy images, as mean intensity and standard deviation maps (seen below the instantaneous image), illustrates a red-coloured area in the mean image, where cavitation is found to be present for the total duration of acquisition; on the contrary, the standard deviation image, having discarded the locations that exhibit zero variations, demonstrates the regions with high variability in intensity values throughout the test case. Three locations of transient behaviour inside the injection hole are identified from the standard deviation image; one (1) approximately at the top mid-length of the hole that is related to the extent of the top entry cavitation structures and the bulk of the void volume located below (diameter-wise) that area. The second (2) location is shortly upstream the exit of the hole and on the top half of the channel diameter, and it is related to detached bubble clouds that are seen to leave the main void and travel along the channel length. The third location (3) with increased deviation from the mean is at the vicinity of the entrance of the channel, where the transient string (or vortex) cavitation phenomenon is captured and illustrated in the contour plots. These structures are vortices that capture vapour (or gas) in their core, appear as thin dark tubes on a shadowgraphy image and have a lifetime of 25-50 $\mu \mathrm{s}$. Moreover, the periodic detachment of vapour bubble clouds from the main cavity that give rise to increased variability in intensity values found in the standard deviation image is a bubble cloud shedding mechanism almost identical to the 
(a)

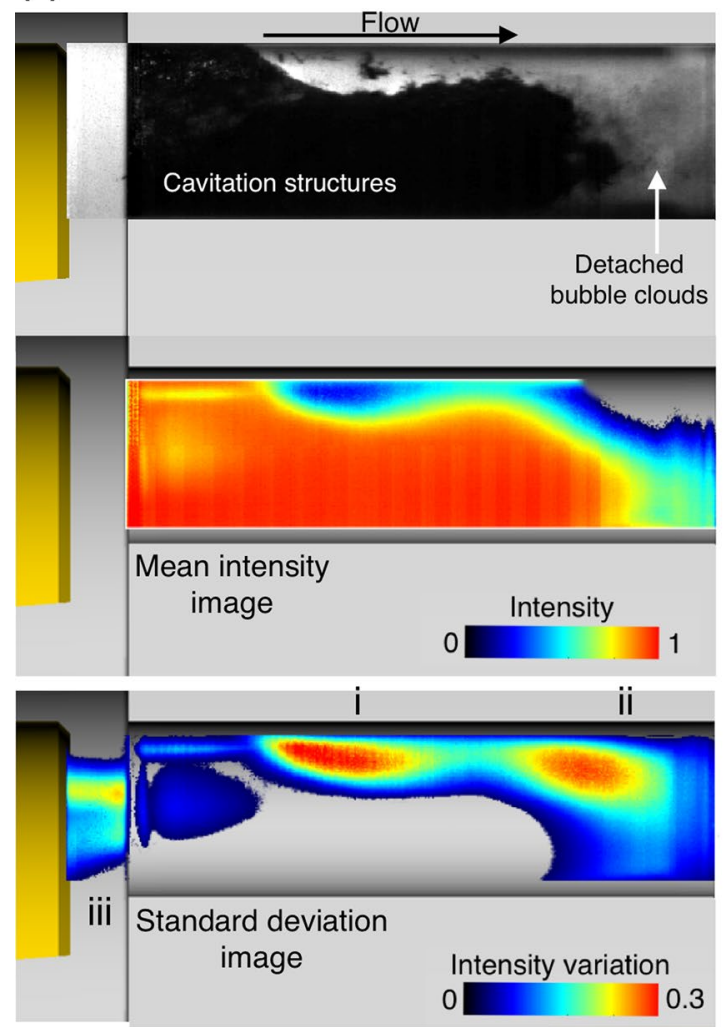

(b)
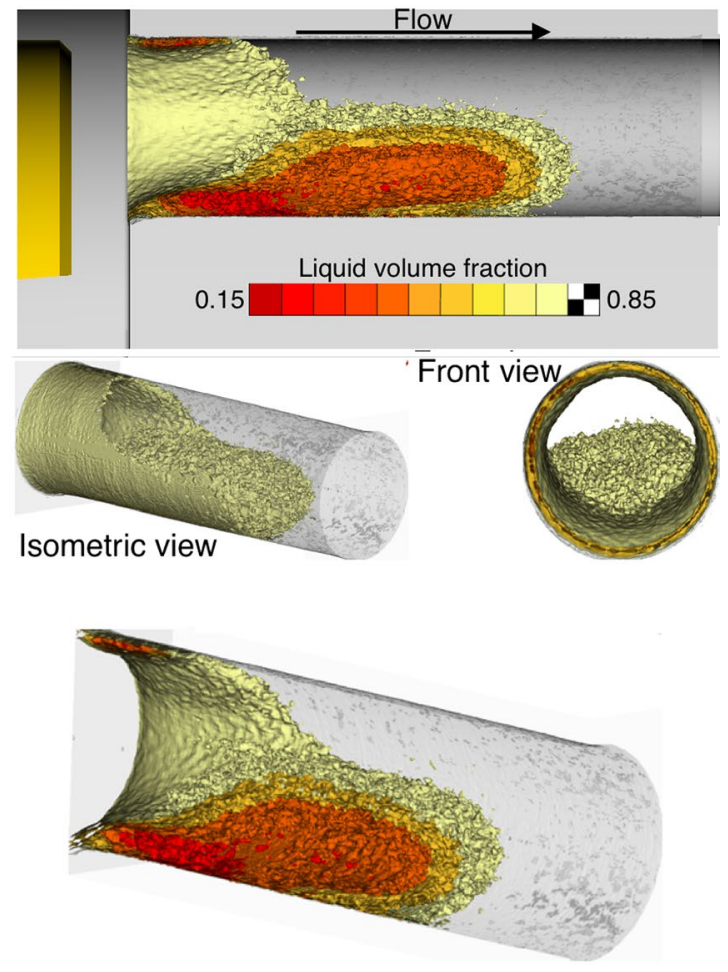

Fig. 4 a Instantaneous flow shadowgraphy image and corresponding mean and standard deviation images false-coloured according to pixel intensity values. b micro-CT measurements of liquid volume frac-

one reported in hydrofoils, i.e., in Choi and Ceccio (2007), Coutier-Delgosha et al. (2006) and Sun et al. (2015).

Focusing only on the visualisation sample of Fig. 4a, an observer may come to a false-representation of cavitation that seems to entirely block the first half of the flow channel. However, observation of the micro-CT results on Fig. 4b, immediately corrects the wrong perception of the flow given previously. The two isometric view representations in Fig. $4 \mathrm{~b}$ correspond to the complete channel, a cut at the symmetry plane as well as the front view of $80 \%$ liquid volume fraction. They demonstrate that cavitation starts as a ring, at the entrance of the nozzle hole, leaving the volume around the axis of the hole available to the liquid flow. As the nozzle model promotes asymmetric flow entry to the orifice, flow velocities and turbulence inside the channel differ from top to bottom. Thus, the cavitation cloud shapes accordingly, as seen at the top micro-CT image of Fig. 4b, where half of the channel is illustrated. Low values of liquid volume fraction are observed at the top of the channel; cavitation diminishes relatively quickly (almost at the first quarter of the hole length). Similar levels of liquid volume fraction at the bottom of the channel extend further inside the flow orifice up to almost $3 / 4$ of its length.

tion presented as side-view-cut iso-surfaces and isometric and front view images. Flow conditions are identical for (a) and (b) $[\mathrm{CN}=2.1$, $R e=55.5 \mathrm{k}, C_{\mathrm{d}}=0.66$ and needle lift of $\left.1 \mathrm{~mm}\right]$

A direct comparison of shadowgraphy versus micro-CT results is illustrated in Fig. 5, where the top row presents an instantaneous, a mean and a standard deviation image of the flow inside the channel at a low cavitation number $(\mathrm{CN})$ of 1.5. At the bottom row of the same figure, micro-CT results of the exact flow conditions are presented as contour plot on the symmetry plane, liquid volume fraction isosurfaces on half-channel and projected vapour volume fraction of the total depth of the channel. In brief, from top left to right, the instantaneous image illustrates a snapshot of all observed flow phenomena inside the flow channel like string cavitation and a detached bubble cloud; the mean image alongside presents a qualitative representation of the vapour cloud and the standard deviation image illustrates the locations with increased variability. On the bottom row, a contour plot of liquid volume fraction on the symmetry plane is displayed on the left, followed by a half-channel representation of liquid fraction iso-surfaces and the third image illustrates projected vapour volume fraction. While a plane-cut contour plot quantifies liquid volume fraction as low as $50 \%$, for the presented conditions, cavitation extent does not look similar to an instantaneous flow image. This discrepancy between shadowgraphy qualitative data and 
(a)

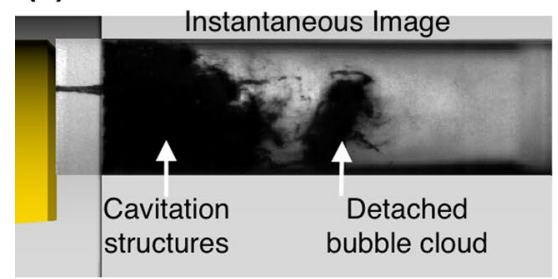

(d)

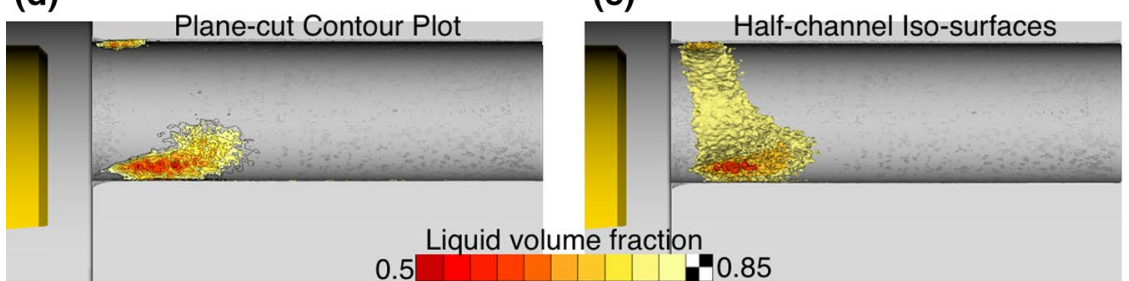

(c)
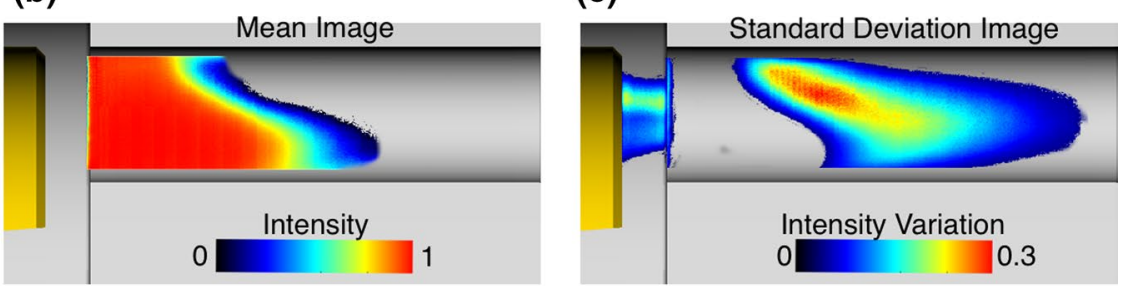

(f)

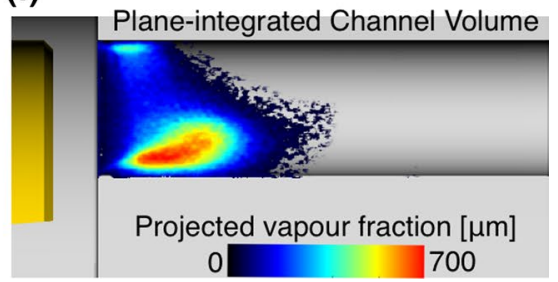

Fig. 5 Comparison of high-speed shadowgraphy images versus micro-CT liquid volume fraction. a Instantaneous shadowgraphy image; b mean cavitation image; $\mathbf{c}$ standard deviation images; d liq- uid volume fraction on the mid-plane; e half-channel liquid fraction iso-surfaces; f plane-integrated channel volume vapour fraction contours $\left[\mathrm{CN}=1.5, R e=55.5 \mathrm{k}, C_{\mathrm{d}}=0.7\right.$ at $1-\mathrm{mm}$ needle lift $]$
micro-CT volume fraction measurements is not minimised even when comparing a half-channel representation with liquid volume fraction iso-surfaces; it is evident from the results that even a liquid volume fraction as high as marginally below $85 \%$ is able of blocking the light intensity, in the case of shadowgraphy. However, comparison of the planeintegrated micro-CT results to imaging data proves to be more realistic, as locations with projected vapour fraction marginally larger than $0 \mu \mathrm{m}$ coincide with that locations of shadowgraphy images that exhibit minimum to zero intensity variability.

\subsection{Liquid fraction measurements}

Quantitative liquid volume fraction measurements can quantify the effect of various operating parameters on cavitation.

Initially, the effect of the needle valve lift is examined in Figs. 6 and 7. In detail, Fig. 6 illustrates a direct comparison of micro-CT data (top and middle rows) versus highspeed shadowgraphy (bottom row). As shown in Fig. 6, the needle valve distance to the orifice entrance varies from 2 to $1 \mathrm{~mm}$; the latter has been selected as the nominal lift for the majority of the test cases. The two presented cases share a cavitation number of $\mathrm{CN}=1.5$ and a Reynolds number that varies between $R e=47$ and $55 \mathrm{k}$, for the increased lift and the lower lift case, respectively. Given the expected negligible effect of marginal differences of the Reynolds number on cavitation structures (Mitroglou et al. 2016), it is evident that presented data are mainly affected by the change in needle valve lift. For the 2-mm needle lift value, the measurements show a confined vapour distribution, where liquid volume fraction values are as high as $80 \%$. As the needle valve lift is reduced to $1 \mathrm{~mm}$ (right-hand side of Fig. 6), the developed phenomena become more intense, compared to the high needle lift case. For the case of $1 \mathrm{~mm}$ lift, liquid volume fraction iso-surfaces denote that cavitation now extends almost up to one-third of the hole length and in the core of the created cavitation cloud, liquid volume fraction values are as low as $60 \%$. Finally, interpretation of the high-speed shadowgraphy standard deviation images at the bottom row of Fig. 6 dictates that the higher the needle lift, the more the cavitation cloud instability inside the flow channel. This is clearly shown by the large red-coloured area that presents a variability of $\sim 30 \%$ from the mean intensity values. However, the opposite trend is captured for the case of string cavitation visible at the vicinity of the hole entrance; the low needle lift of $1 \mathrm{~mm}$ shows increased variability compared to the high needle lift of $2 \mathrm{~mm}$.

At even lower needle lifts than $1 \mathrm{~mm}$, a similar trend is found and is depicted in Fig. 7, where the nominal lift of $1 \mathrm{~mm}$ is compared against the lowest tested lift of $0.5 \mathrm{~mm}$. The flow conditions in both cases are similar sharing the same cavitation number of $\mathrm{CN}=2.1$ and $R e=55 \mathrm{k}$ for the $1-\mathrm{mm}$ lift case and $R e=36 \mathrm{k}$ for the lowest lift case. This difference in Reynolds numbers is due to the low lift that inevitably limits the flow area available upstream the channel entrance to values lower than the channel's crosssectional area. Nevertheless, the cavitation number of 2.1 causes extended cavitation in the 1-mm lift case, as shown by the contour plot on the channel's symmetry plane; however, as shown in the embedded front view image, there are still locations inside the channel that exhibit either negligible, or no cavitation at all. Despite the lower Reynolds 


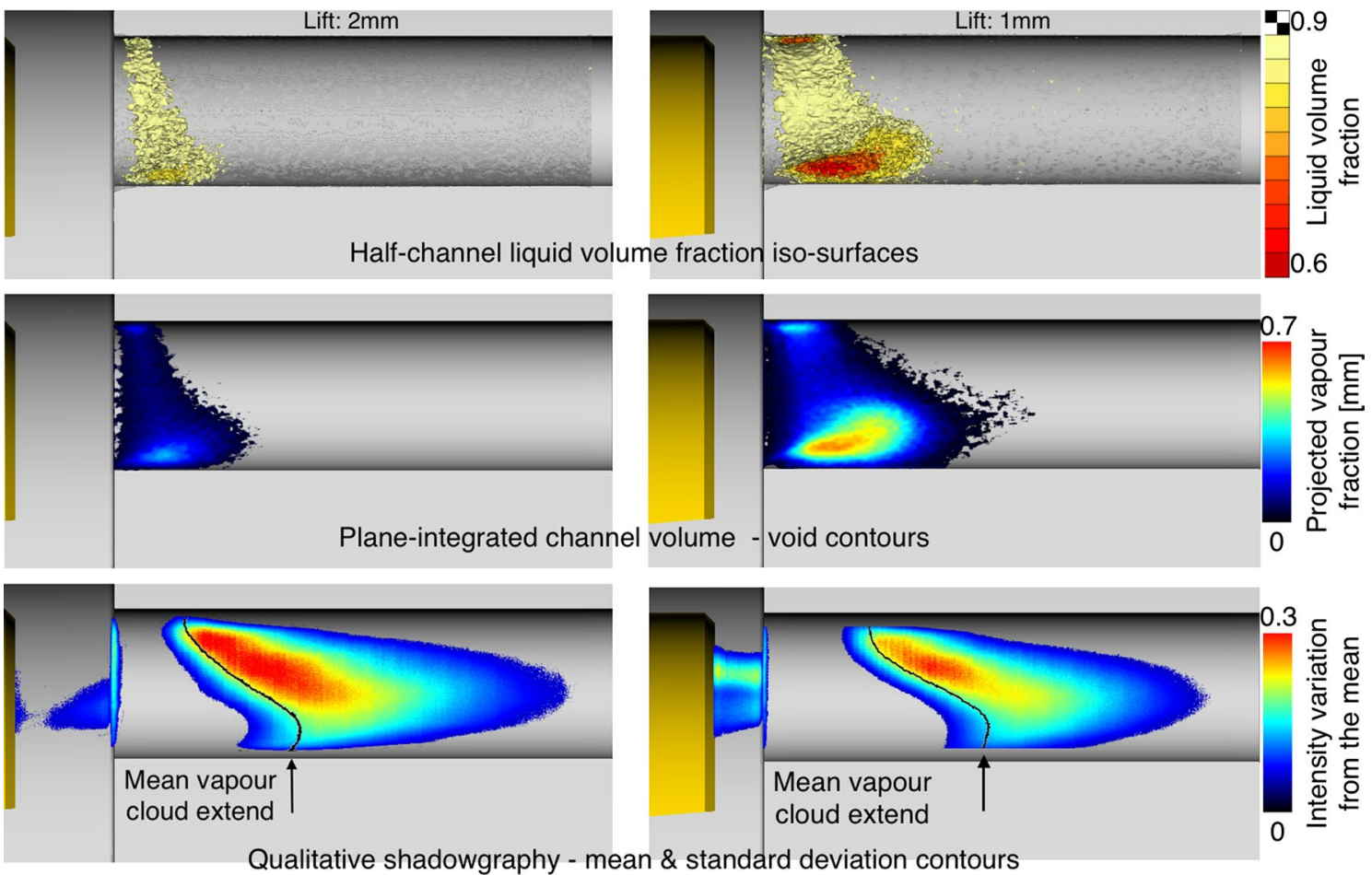

Fig. 6 Effect of needle lift position on vapour pockets presented as liquid fraction iso-surfaces for half the channel. Cavitation number is fixed at 1.5 and Reynolds number equals to $47 \mathrm{k}$ for needle lift $=2 \mathrm{~mm}$ and $55.5 \mathrm{k}$ for the low needle lift of $1 \mathrm{~mm}$

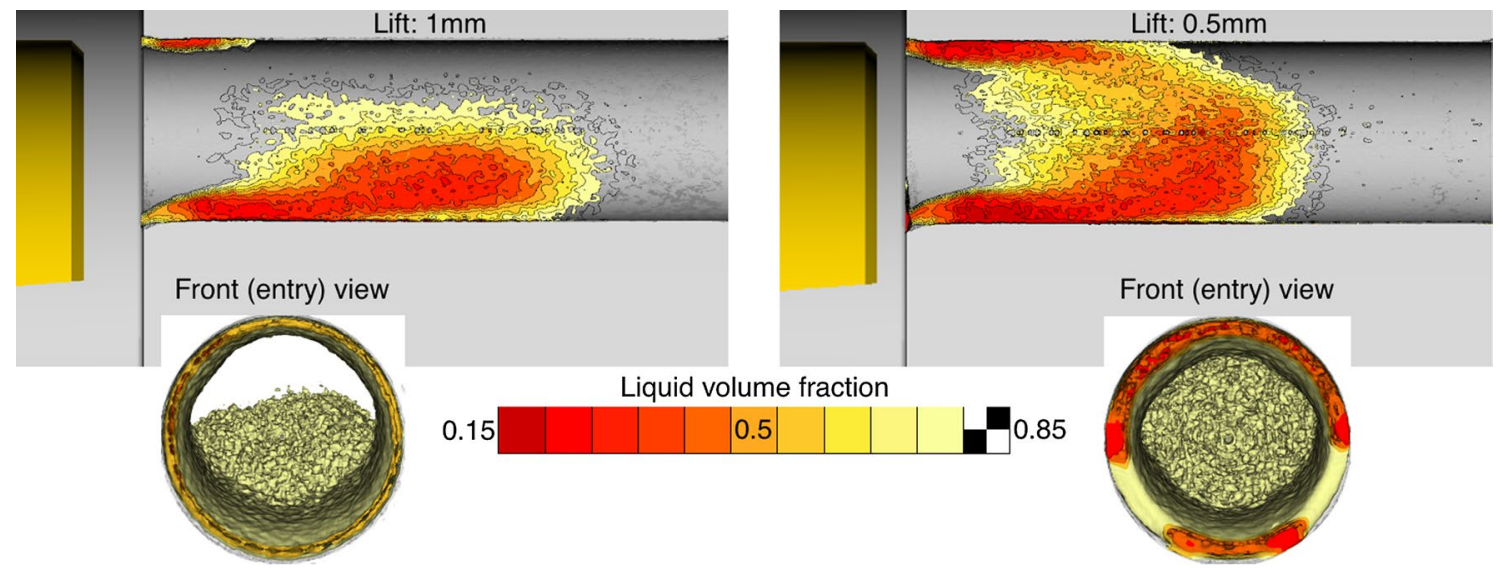

Fig. 7 Effect of needle lift position on vapour pockets presented as liquid fraction contours on the channel symmetry plane. Cavitation number is fixed at 2.1 and Reynolds number is $55.5 \mathrm{k}$ for the high lift of $1 \mathrm{~mm}$ and $36.5 \mathrm{k}$ for the low lift value of $0.5 \mathrm{~mm}$

number for the lowest lift case of $0.5 \mathrm{~mm}$, the flow picture on the symmetry plane is drastically different to the one of $1 \mathrm{~mm}$ lift. It is evident from both illustrated views of $0.5 \mathrm{~mm}$ lift that almost $1 / 3$ of the channel length is occupied by liquid volume fraction ranging from 15 to $60 \%$. Additionally, the area exhibiting extra low liquid volume fraction of $\sim 15 \%$ is larger for the lowest lift case, compared to the basic lift of $1 \mathrm{~mm}$. Finally, it has been established that needle valve lift has a pronounced effect on cavitation structures, as it promotes turbulence, which, in turn, promotes cavitation. Unfortunately, the total channel blockage with vapour at the lowest needle lift prevented meaningful shadowgraphy data to be collected, as there was no light penetration through the channel due to increased vapour.

A direct comparison of the effect of cavitation number $(\mathrm{CN})$ on vapour generation is illustrated in Fig. 8. At the nominal needle lift of $1 \mathrm{~mm}$, Fig. 8a corresponds to $\mathrm{CN}=2.1$ and it presents, from top to bottom, micro-CT data in the form of 
half-channel iso-surfaces, plane-integrated channel volume void fraction and hi-speed shadowgraphy standard deviation images; the same data representation is illustrated in Fig. 8b for the lower cavitation number of $\mathrm{CN}=1.5$. The Reynolds number for both cases is fixed at $R e=55 \mathrm{k}$. It is immediately evident that increasing $\mathrm{CN}$ has a significant effect on the cavitation cloud extent; in Fig. 8a, the vapour cloud extends up to $75 \%$ of the channel length and liquid fraction in its core is as low as 5\%. Instead, in Fig. 8b, a limited extent of cavitation structures is shown and liquid volume fraction as low as $55 \%$. The observed differences in vapour production inside the flow channel were expected, since the pressure difference across the injection hole, as denoted by $\mathrm{CN}$, is mainly responsible for the initiation and development of geometric cavitation. However, what was unexpected in these illustrations is a slight vortex-like vapour shape that appears above the axis of symmetry of the channel at the left-hand side of the cavitation cloud of $\mathrm{CN}=2.1$ case (marked inside an ellipse). It resembles a string-like shape with its tip facing the hole entrance. This is the vicinity where cavitation strings have been recorded by high-speed shadowgraphy experiments as it is verified in the bottom row standard deviation images, where variability is indeed increased at the hole entrance vicinity. Of course, it is impossible for the time-averaged data of microCT to capture such a transient feature; however, the existence of the previously described shape may be closely related to increased vorticity in that location, which may be adequate to promote string formation. Finally, the same structures exist also in the lower $\mathrm{CN}$ case of 1.5 (bottom image of Fig. 8b); however, their intensity and lifetime is not as pronounced as at higher $\mathrm{CN}$.

Finally, Fig. 9 illustrates the effect of Reynolds number on cavitation development. At a fixed $\mathrm{CN}=1.5$ and needle lift of $1 \mathrm{~mm}$, it is evident that an increase in Reynolds number from $35 \mathrm{k}$ (Fig. 9a) to $55 \mathrm{k}$ (Fig. 9b) has a marginal effect on vapour pockets inside the flow channel; increasing $R e$ from 35 to $55 \mathrm{k}$ only resulted in marginally lower liquid volume fraction measured in the core of the vapour cloud and in marginally larger channel volume occupied by the highest void fractions (middle set of images in Fig. 9), compared to $R e=35 \mathrm{k}$. It should be noted that the false colour scale for the Reynolds number comparison ranges from 0.6 to 0.9, as opposed to the left-hand side of Fig. 8, where it ranges from 0.15 to 0.85 ; hence, the different contour colours for the case of $\mathrm{CN}=1.5$ and $R e=55 \mathrm{k}$, which is identical in both columns of this figure. Finally, the transient behaviour of both cases is presented in the standard deviation shadowgraphy images (bottom row of Fig. 9), where low Reynolds number showed increased variability in cavitation cloud shapes inside the channel, while the high Reynolds number demonstrated increased string cavitation activity at the entrance of the hole. (a)
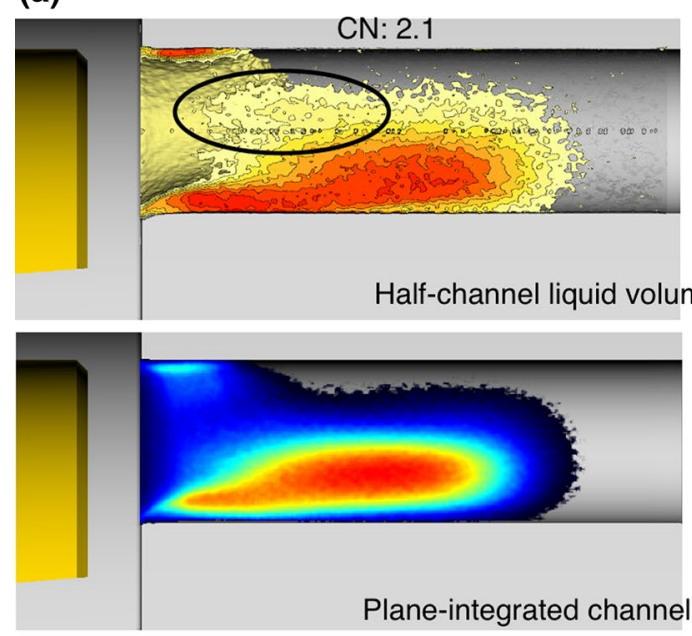

Half-channel liquid volume

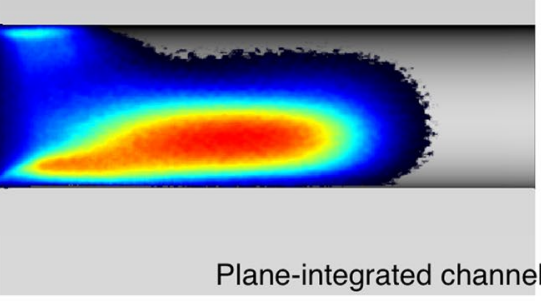

Plane-integrated channel

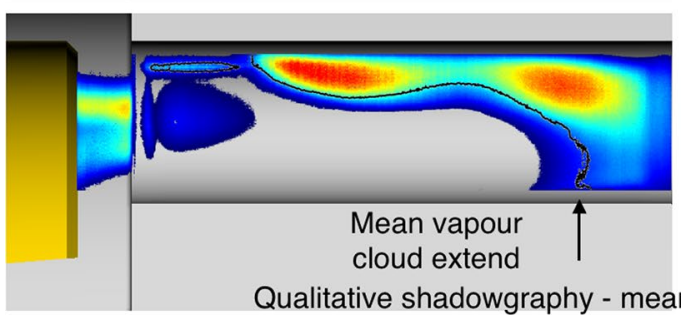

(b)
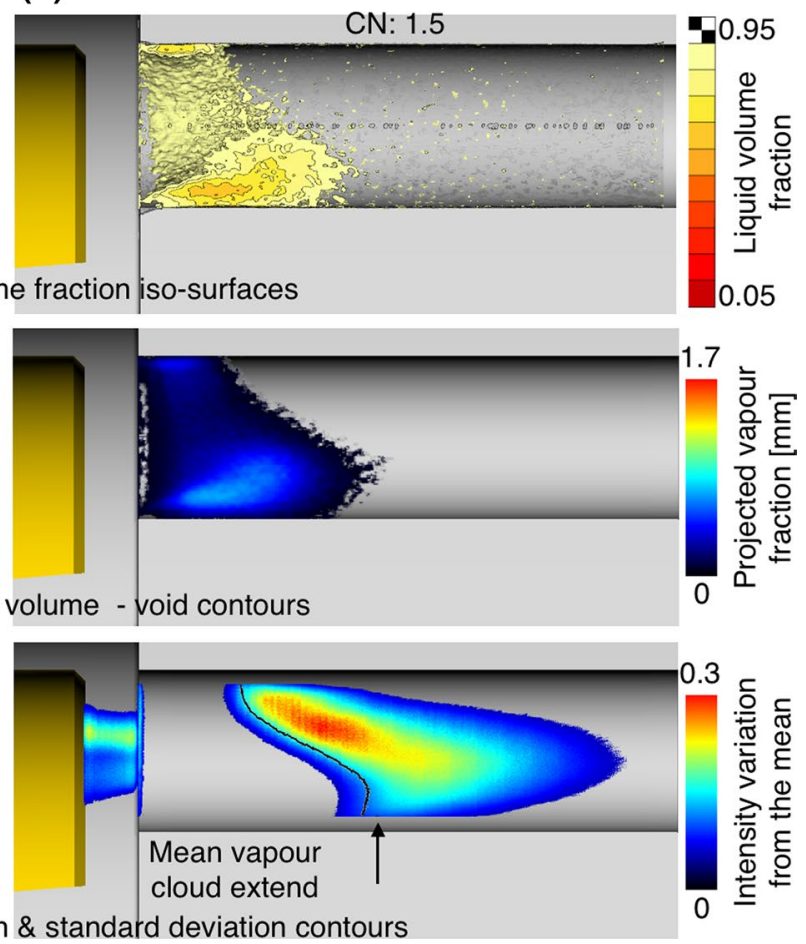

oid contours
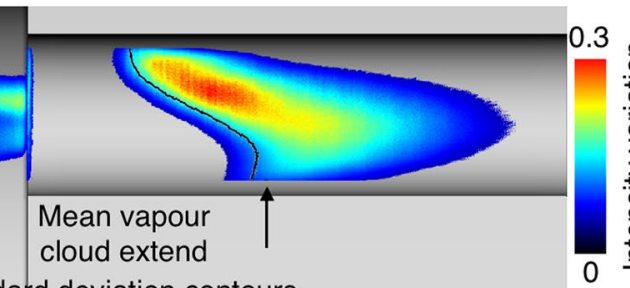

Fig. 8 Effect of cavitation number $(\mathrm{CN})$ on vapour pockets at fixed needle lift of $1 \mathrm{~mm}$ and $\operatorname{Re}$ of $55 \mathrm{k} ; \mathbf{a} \mathrm{CN}=2.1$ and $\mathbf{b} \mathrm{CN}=1.5$ 
(a)

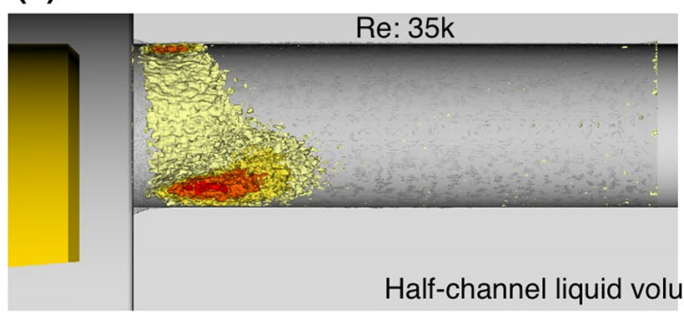

(b)
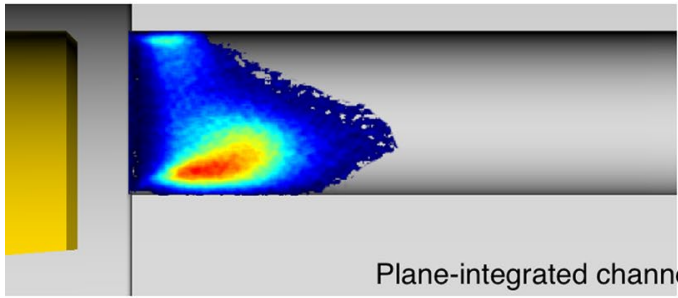

Plane-integrated channel volume - void contours

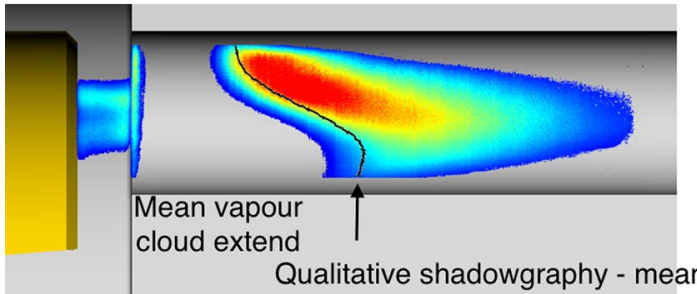

Qualitative shadowgraphy - mean \& standard deviation contours
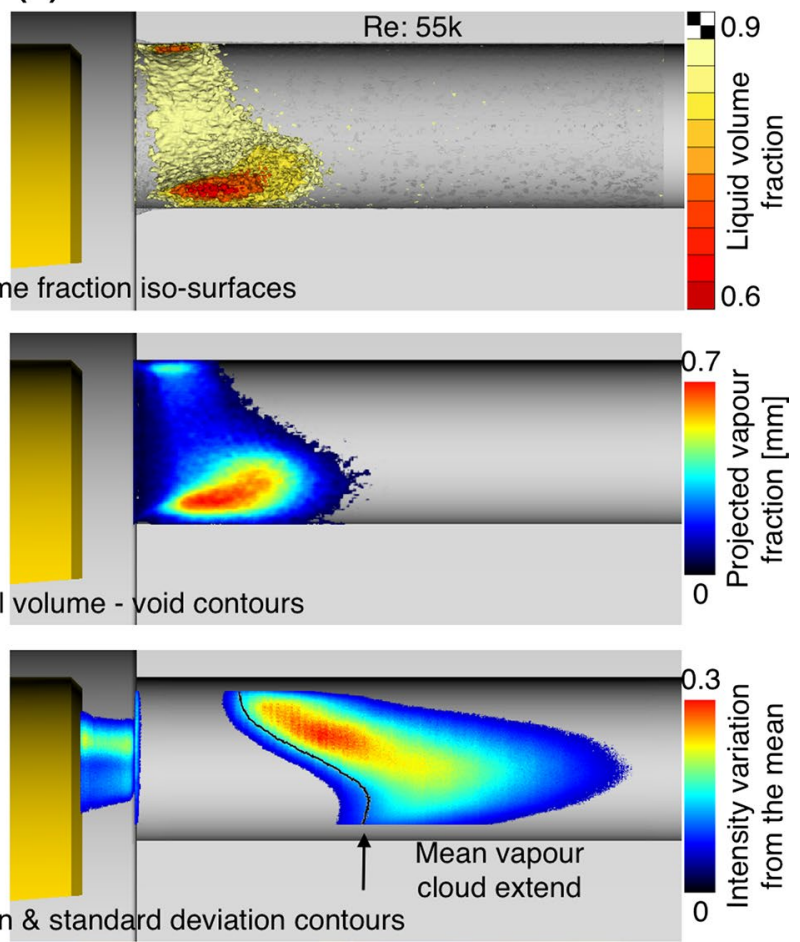

Fig. 9 Effect of Reynolds number on vapour pockets at a fixed lift of $1 \mathrm{~mm}$ and cavitation number of 1.5; a $R e: 35 \mathrm{k}$ and $\mathbf{b} R e: 55 \mathrm{k}$

\section{Conclusions}

The present study investigated the possibility of applying the micro-CT technique on highly transient cavitating diesel fuel flows and results appear promising in terms of time-averaged quantitative fuel vapour measurements. The technique produced results that compared seamlessly to the high-speed visualisation data, obtained from an identical transparent model. It has been established that a combination of both techniques is advantageous, as it provides a mix of average quantitative measurements and instantaneous qualitative images that provide valuable statistical information on the transient features of the flow field. The effect of flow parameters on cavitation structures has been quantified, and the expected results were observed. Needle lift valve was found to have a strong effect on vapour generation and vapour pocket extent inside the channel. Moreover, cavitation number effects on cavitation behaviour was examined and the expected results were confirmed. Finally, the effect of Reynolds number was investigated; however, the results do not show any significant change in cavitation vapour cloud behaviour.

Acknowledgements The research leading to these results has received funding from the People Programme (IOF Marie Curie Actions) of the European Union's Seventh Framework Programme FP7/2007-2013/under REA Grant Agreement No. 300410.
Open Access This article is distributed under the terms of the Creative Commons Attribution 4.0 International License (http://creativecommons.org/licenses/by/4.0/), which permits unrestricted use, distribution, and reproduction in any medium, provided you give appropriate credit to the original author(s) and the source, provide a link to the Creative Commons license, and indicate if changes were made.

\section{References}

Andriotis A, Gavaises M, Arcoumanis C (2008) Vortex flow and cavitation in diesel injector nozzles. J Fluid Mech 610:195-215. doi: $10.1017 /$ S0022112008002668

Arcoumanis C, Flora H, Gavaises M, Badami M (2000) Cavitation in real-size multi hole diesel injector nozzles. SAE technical paper 2000-01-1249. doi: 10.4271/2000-01-1249

Badock C, Wirth R, Fath A, Leipertz A (1999) Investigation of cavitation in real size diesel injection nozzles. Int $\mathrm{J}$ Heat Fluid Flow 20(5):538-544

Battistoni M, Duke DJ, Swantek AB, Tilocco FZ, Powell CF, Som S (2015) Effects of noncondensable gas on cavitating nozzles. Atomization Sprays 25(6):453-483

Bauer D, Chaves H, Arcoumanis C (2012) Measurements of void fraction distribution in cavitating pipe flow using X-ray CT. Meas Sci Technol 23(5):055302. doi:10.1088/0957-0233/23/5/055302

Bergwerk W (1959) Flow pattern in diesel nozzle spray holes. Proc Inst Mech Eng 173(1):655-660

Berrocal E, Kristensson E, Richter M, Linne M, Aldén M (2008) Application of structured illumination for multiple scattering suppression in planar laser imaging of dense sprays. Opt Express 16(22):17870-17881 
Blessing M, Konig G, Kruger C, Michels U, Schwarz V (2003) Analysis of flow and cavitation phenomena in diesel injection nozzles and its effects on spray and mixture formation. In: Fuel injection systems 2003: IMechE conference transactions 2003, vol 2. p 21

Chaouki J, Larachi F, Dudukovic MP (1997) Noninvasive tomographic and velocimetric monitoring of multiphase flows. Ind Eng Chem Res 36(11):4476-4503. doi:10.1021/ie970210t

Chaves H, Knapp M, Kubitzek A, Obermeier F, Schneider T (1995) Experimental study of cavitation in the nozzle hole of diesel injectors using transparent nozzles. SAE technical paper 950290. doi: $10.4271 / 950290$

Choi J, Ceccio SL (2007) Dynamics and noise emission of vortex cavitation bubbles. J Fluid Mech 575:1. doi:10.1017/ s0022112006003776

Coutier-Delgosha O, Devillers JF, Pichon T, Vabre A, Woo R, Legoupil S (2006) Internal structure and dynamics of sheet cavitation. Phys Fluids (1994-present) 18(1):017103. doi: $10.1063 / 1.2149882$

Du D-X, Beni AN, Farajzadeh R, Zitha PL (2008) Effect of water solubility on carbon dioxide foam flow in porous media: an X-ray computed tomography study. Ind Eng Chem Res 47(16):6298-6306

Dudukovic MP (2000) Opaque multiphase reactors: experimentation, modeling and troubleshooting. Oil Gas Sci Technol 55(2): $135-158$

Duke DJ (2015) A review of synchrotron radiation diagnostics for fluid mechanics. In: Proceedings of the 7th Australian conference on laser diagnostics in fluid mechanics and combustion. Melbourne, pp 15-22

Duke DJ, Kastengren AL, Tilocco FZ, Swantek AB, Powell CF (2013) X-ray radiography measurements of cavitating nozzle flow. Atomization Sprays 23(9):841-860

Duke DJ, Kastengren AL, Swantek AB, Sovis N, Fezzaa K, Neroorkar K, Schmidt DP (2014) Comparing simulations and X-ray measurements of a cavitating nozzle. In: Proceedings of the ilassamericas 26th annual conference on liquid atomization and spray systems. Portland

Duke D, Swantek A, Kastengren A, Fezzaa K, Powell C (2015a) Recent developments in X-ray diagnostics for cavitation. SAE Int J Fuels Lubr 8(1):135-146. doi:10.4271/2015-01-0918

Duke DJ, Swantek AB, Kastengren AL, Powell CF (2015b) X-ray diagnostics for cavitating nozzle flow. J Phys: Conf Ser 656:012110. doi:10.1088/1742-6596/656/1/012110

Dular M, Petkovšek M (2015) On the mechanisms of cavitation erosion-coupling high speed videos to damage patterns. Exp Thermal Fluid Sci 68:359-370. doi:10.1016/j. expthermflusci.2015.06.001

Feldkamp LA, Davis LC, Kress JW (1984) Practical cone-beam algorithm. JOSA A 1(6):612-619

Ganesh H, Mäkiharju SA, Ceccio SL (2015) Interaction of a compressible bubbly flow with an obstacle placed within a shedding partial cavity. In: Journal of physics: conference series, vol 656. p 012151)

Gavaises M, Andriotis A, Papoulias D, Mitroglou N, Theodorakakos A (2009) Characterization of string cavitation in large-scale diesel nozzles with tapered holes. Phys Fluids 21(5):052107. doi:10.1063/1.3140940

Grohse EW (1955) Analysis of gas-fluidized solid systems by X-ray absorption. AIChE J 1(3):358-365

Hayashi T, Suzuki M, Ikemoto M (2013) Effects of internal flow in a diesel nozzle on spray combustion. Int J Engine Res 14(6):646654. doi:10.1177/1468087413494910

Heindel TJ (2011) A review of X-ray flow visualization with applications to multiphase flows. J Fluids Eng 133(7):074001. doi: $10.1115 / 1.4004367$
Ikeda T, Kotani K, Maeda Y, Kohno H (1983) Preliminary study on application of X-ray CT scanner to measurement of void fractions in steady state two-phase flows. J Nucl Sci Technol 20(1):1-12. doi:10.1080/18811248.1983.9733354

Jollet F, Heilig A, Bitner K, Niemeyer D, Dinkelacker F (2013) Comparison of experiments and numerical simulations of high pressure transparent injection nozzles. In: Proceedings of the 25th European conference on liquid atomization and spray systems. Chania, pp 462-468

Kak AC, Slaney M (2001) Principles of computerized tomographic imaging. Class Appl Math. doi:10.1137/1.9780898719277

Kastengren A, Powell CF (2014) Synchrotron X-ray techniques for fluid dynamics. Exp Fluids. doi:10.1007/s00348-014-1686-8

Kastengren A, Powell CF, Arms D, Dufresne EM, Gibson H, Wang J (2012) The 7BM beamline at the APS: A facility for timeresolved fluid dynamics measurements. J Synchrotron Radiat 19(Pt 4):654-657. doi:10.1107/S0909049512016883

Ketcham RA, Carlson WD (2001) Acquisition, optimization and interpretation of X-ray computed tomographic imagery: applications to the geosciences. Comput Geosci 27(4):381-400

Koukouvinis P, Gavaises M, Li J, Wang L (2016) Large eddy simulation of diesel injector including cavitation effects and correlation to erosion damage. Fuel 175:26-39. doi:10.1016/j.fuel.2016.02.037

Leick P, Grzeszik R, Arndt S, Wissel S (2011) Suppression of multiple scattering using structured light sheets-A first assessment for diesel and gasoline spray visualization. In: Proceedings of the 24th annual ILASS-Europe conference. Estoril

Lin K-C, Carter C, Kastengren A, Fezzaa K (2012) Exploration of aerated-liquid jets using X-ray phase contrast imaging and X-ray radiography. In: Proceedings of 12 th triennial international conference on liquid atomization and spray systems. Heidelberg, p 8

Linne M, Paciaroni M, Hall T, Parker T (2006) Ballistic imaging of the near field in a diesel spray. Exp Fluids 40(6):836-846

Mäkiharju SA, Gabillet C, Paik B-G, Chang NA, Perlin M, Ceccio SL (2013) Time-resolved two-dimensional X-ray densitometry of a twophase flow downstream of a ventilated cavity. Exp Fluids 54(7):1-21

Marashdeh Q, Fan L-S, Du B, Warsito W (2008) Electrical capacitance tomography-a perspective. Ind Eng Chem Res 47(10):3708-3719. doi:10.1021/ie0713590

Miranda R, Chaves H, Martin U, Obermeier F (2003) Cavitation in a transparent real size VCO injection nozzle. In: Proceedings of ICLASS. Sorrento

Mitroglou N, Gavaises M (2013) Mapping of cavitating flow regimes in injectors for medium/heavy duty diesel engines. Int $\mathbf{J}$ Engine Res 14(6):590-605. doi:10.1177/1468087413500491

Mitroglou N, Gavaises M, Arcoumanis C (2011) Cavitation inside enlarged and real-size fully transparent injector nozzles and its effect on near nozzle spray formation. In: Droplet impact phenomena and spray investigations workshop 2011. Bergamo

Mitroglou N, Gavaises M, Arcoumanis C (2012) Spray stability from VCO and a new diesel nozzle design concept. In: Proceedings of the IMechE conference on fuel systems for IC engines. IMechE, WOODHEAD PUBL LTD, London, pp 279-290

Mitroglou N, McLorn M, Gavaises M, Soteriou C, Winterbourne M (2014) Instantaneous and ensemble average cavitation structures in diesel micro-channel flow orifices. Fuel 116:736-742. doi:10.1016/j.fuel.2013.08.060

Mitroglou N, Lorenzi M, Santini M, Gavaises M, Assanis D (2015) Application of cone-beam micro-ct on high-speed diesel flows and quantitative cavitation measurements. In: Journal of physics: conference series, vol 656. doi:10.1088/1742-6596/656/1/012094

Mitroglou N, Lorenzi M, Santini M, Gavaises M (2016) X-ray microcomputed tomography for cavitating diesel nozzles. In: Proceedings of the 27th annual conference on liquid atomization and spray systems. Brighton 
Moon S, Liu Z, Gao J, Dufresne E, Fezzaa K, Wang J (2010) Ultrafast $\mathrm{X}$-ray phase-contrast imaging of high-speed fuel sprays from a two-hole diesel nozzle. In: ILASS Americas, 22nd annual conference on liquid atomization and spray systems. Cincinnati

Moon S, Komada K, Sato K, Yokohata H, Wada Y, Yasuda N (2015) Ultrafast X-ray study of multi-hole GDI injector sprays: effects of nozzle hole length and number on initial spray formation. Exp Thermal Fluid Sci 68:68-81. doi:10.1016/j. expthermflusci.2015.03.027

Nurick WH (1976) Orifice cavitation and its effect on spray mixing. J Fluids Eng 98(4):681-687

Payri F, Arrègle J, López J, Hermens S (2006) Effect of cavitation on the nozzle outlet flow, spray and flame formation in a diesel engine, SAE technical paper 2006-01-1391. doi: 10.4271/2006-01-1391

Potz D, Christ W, Dittus B (2000) Diesel nozzle-The determining interface between injection system and combustion chamber. In: Proceedings of the THIESEL international conference on thermo-and fluid dynamic processes in diesel engines. Valencia

Powell CF, Yue Y, Poola R, Wang J (2000) Time-resolved measurements of supersonic fuel sprays using synchrotron X-rays. J Synchrotron Radiat 7(Pt 6):356-360. doi:10.1107/ S0909049500013431

Reid BA, Hargrave GK, Garner CP, Wigley G (2010) An investigation of string cavitation in a true-scale fuel injector flow geometry at high pressure. Phys Fluids 22(3):031703. doi:10.1063/1.3372174

Reid BA, Gavaises M, Mitroglou N, Hargrave GK, Garner CP, Long EJ, McDavid RM (2014) On the formation of string cavitation inside fuel injectors. Exp Fluids 55(1662):1-8. doi:10.1007/ s00348-013-1662-8

Roth H, Gavaises M, Arcoumanis C (2002) Cavitation initiation, its development and link with flow turbulence in diesel injector nozzles. SAE technical paper 2002-01-0214. doi:10.4271/2002-01-0214

Roth H, Giannadakis E, Gavaises M, Arcoumanis C, Yanagihara H, Sakata I (2005) Effect of multi-injection strategy on cavitation development in diesel injector nozzle holes. SAE technical paper 2005-01-1237

Rowe PN, Partridge BA (1997) An X-ray study of bubbles in fluidised beds. Chem Eng Res Des 75:S116-S134

Santini M, Guilizzoni M (2014) 3D X-ray micro computed tomography on multiphase drop interfaces: From biomimetic to functional applications. Colloids Interface Sci Commun 1:14-17
Santini M, Guilizzoni M, Fest-Santini S (2013) X-ray computed microtomography for drop shape analysis and contact angle measurement. J Colloid Interface Sci 409:204-210. doi:10.1016/j.jcis.2013.06.036

Santini M, Guilizzoni M, Fest-Santini S, Lorenzi M (2015) A novel technique for investigation of complete and partial anisotropic wetting on structured surface by X-ray microtomography. Rev Sci Instrum 86(2):023708. doi:10.1063/1.4908171

Soteriou C, Andrews R, Smith M (1995) Direct injection diesel sprays and the effect of cavitation and hydraulic flip on atomization. SAE technical paper 950080. doi: 10.4271/950080

Soteriou C, Lambert M, Zuelch S, Passerel D (2006) The flow characteristics of high efficiency diesel nozzles with enhanced geometry holes. In: Proceedings of the THIESEL international conference on thermo-and fluid dynamic processes in diesel engines. Valencia

Stutz B, Legoupil S (2003) X-ray measurements within unsteady cavitation. Exp Fluids 35(2):130-138

Suh HK, Lee CS (2008) Effect of cavitation in nozzle orifice on the diesel fuel atomization characteristics. Int $\mathrm{J}$ Heat Fluid Flow 29(4):1001-1009. doi:10.1016/j.ijheatfluidflow.2008.03.014

Sun T, Ganesh H, Ceccio S (2015) X-ray densitometry based void fraction flow field measurements of cavitating flow in the wake of a circular cylinder. Bull Am Phys Soc 60

Tang J, Pischinger S, Lamping M, Körfer T et al (2009) Coking phenomena in nozzle orifices of Dl-diesel engines. SAE Int J Fuels Lubr 2(1):259-272. doi:10.4271/2009-01-0837

Walther J (2002) Quantitative Untersuchungen der Innenströmung in kavitierenden Dieseleinspritzdüsen. Ph.D. Thesis, TU Darmstadt. http://tuprints.ulb.tu-darmstadt.de/241/

Wang Y, Liu X, Im K-S, Lee W-K, Wang J, Fezzaa K, Winkelman JR (2008) Ultrafast X-ray study of dense-liquid-jet flow dynamics using structure-tracking velocimetry. Nat Phys 4(4):305-309

Winklhofer E, Kull E, Kelz E, Morozov A (2001) Comprehensive hydraulic and flow field documentation in model throttle experiments under cavitation conditions. In: Proceedings of the ILASS-Europe conference. Zurich, pp 574-579

Yates J, Cheesman D, Lettieri P, Newton D (2002) X-ray analysis of fluidized beds and other multiphase systems. KONA Powder Part J 20:133-143. doi:10.14356/kona.2002016 